\title{
Study on the Effect of Soft and Hard Coal Pore Structure on Gas Adsorption Characteristics
}

\author{
Xun Zhao $\mathbb{D}^{1,2,3}$ Tao Feng, ${ }^{1,2}$ Ping Wang, ${ }^{1,2}$ and Ze Liao ${ }^{1,2}$ \\ ${ }^{1}$ School of Resource, Environment and Safey Engineering, Hunan University of Science and Technology, Xiangtan 411201, China \\ ${ }^{2}$ Work Safety Key Lab on Prevention and Control of Gas and Roof Disasters for Southern Coal Mines, \\ Hunan University of Science and Technology, Xiangtan 411201, Hunan, China \\ ${ }^{3}$ Guizhou Coal Mine Design Research Institute Co.Ltd., Guiyang 550025, China \\ Correspondence should be addressed to Xun Zhao; 247651516@qq.com
}

Received 9 April 2021; Accepted 28 May 2021; Published 12 June 2021

Academic Editor: Zhijie Zhu

Copyright (C) 2021 Xun Zhao et al. This is an open access article distributed under the Creative Commons Attribution License, which permits unrestricted use, distribution, and reproduction in any medium, provided the original work is properly cited.

\begin{abstract}
In order to grasp the effect of soft and hard coal pore structure on gas adsorption characteristics, based on fractal geometry theory, low-temperature nitrogen adsorption and constant temperature adsorption test methods are used to test the pore structure characteristics of soft coal and its influence on gas adsorption characteristics. We used box dimension algorithm to measure the fractal dimension and distribution of coal sample microstructure. The research results show that the initial nitrogen adsorption capacity of soft coal is greater than that of hard coal, and the adsorption hysteresis loop of soft coal is more obvious than that of hard coal. And the adsorption curve rises faster in the high relative pressure section. The specific surface area and pore volume of soft coal are larger than those of hard coal. The number of pores is much larger than that of hard coal. In particular, the superposition of the adsorption force field in the micropores and the diffusion in the mesopores enhance the adsorption potential of soft coal. Introducing the concept of adsorption residence time, it is concluded that more adsorption sites on the surface of soft coal make the adsorption and residence time of gas on the surface of soft coal longer. Fractal characteristics of the soft coal surface are more obvious. The saturated adsorption capacity of soft coal and the rate of reaching saturation adsorption are both greater than those of hard coal. The research results of this manuscript will provide a theoretical basis for in-depth analysis of the adsorption/desorption mechanism of coalbed methane in soft coal seams and the formulation of practical coalbed methane control measures.
\end{abstract}

\section{Introduction}

Coal is a kind of porous solid medium with developed pore structure. The abundant pore structure in the coal body is not only a place for gas storage, but also a channel for gas production. The adsorption state of gas in coal makes the mechanical properties of gas-containing coal different from non-adsorption media. Gas-containing coal is a multi-phase porous medium with temporal and spatial variability, which is composed of pore fluid, matrix pores and micro-fractures. The distribution and connectivity of coal pore directly affect the migration and diffusion of gas. In the process of coal seam mining or gas drainage, the coal seamstress field, fissure field, and gas seepage field change. Fracture expansion and penetration of coal and rock mass caused by mining unloading will change the interaction between coal and gas.

The natural pore and fissure structural characteristics of coal determine that coal has good gas adsorption capacity and storage performance. In recent years, scholars have conducted a lot of research on coal pore structure and gas adsorption characteristics. Bing et al. [1] studied the pore structure and adsorption characteristics of outburst coal and found that micropores with a pore size of 3-5 $\mathrm{nm}$ in outburst coal are the main gas adsorption space. Xue et al. [2] used a variety of test methods to study and found that for the same coal grade, as the degree of structural deformation increases, open pores gradually transform into fine bottleneck pores, 
and the adsorption capacity increases, but the coal porosity decreases. Yuan et al. [3, 4] used nitrogen adsorption method and mercury injection method to study the pore structure of coal and obtained the pore characteristics of coal adsorption and migration; Sitprasert et al. [5] used multiscale methods to study physical adsorption in micropores; Rigby et al. [6] studied the gas adsorption law of irregular meshes. Zhang et al. [7] used the low-temperature liquid nitrogen method to study the influence of structural deformation on the nano-scale pore structure of coal and found that ductile deformed coal has more complex pore structure and a higher fractal dimension than brittle deformed coal. An et al. [8] conducted a fractal study on the pore characteristics of high coal rank coals and found that the pore fractal dimension is negatively correlated with coal metamorphism, porosity, macropore content, and volume median pore size, and has a negative correlation with mesopore content and adsorption pore content. Mercury removal efficiency and ash yield are positively correlated. Jiang et al. [9] used the mercury intrusion method to determine the characteristic parameters of the ultrafine pore structure of coal and found that as the hardness of the coal increases, the fractal dimension of pores continues to decrease. Wang et al. [10] used small-angle rays combined with scanning electron microscopy to find that coal pores are mostly spheres at low coal ranks, and generally ellipsoids with high coal ranks. Klimenko et al. [11] used the conditional moment model to study the fractal characteristics of the transportation, storage, and adsorption processes in the pores of $\mathrm{CO}_{2}$ porous media. However, there are relatively few systematic studies on the pore characteristics of soft coal bodies. A large number of studies have shown that the pore distribution of porous media satisfies self-similarity and conforms to the fractal law $[12,13]$. Harpalant et al. [14-18] showed that the coal body will produce expansion stress and deformation after adsorbing gas. There are few systematic research results on the influence of soft coal pore structure on gas adsorption performance.

A typical soft coal body has a smaller consolidation coefficient and a larger initial dispersion rate. This manuscript takes typical soft coal seams and contrasting hard coals as the research objects. Using scanning electron microscope, low-temperature nitrogen adsorption test and isothermal adsorption test, combined with fractal geometry theory, the pore structure characteristics of soft coal and hard coal and their influence on gas adsorption characteristics are studied. In order to quantitatively characterize the pore distribution characteristics of soft coal and hard coal, the mechanical properties of soft coal and the gas flow law in soft coal are studied. The research results of this manuscript will provide a theoretical basis for in-depth analysis of the adsorption/ desorption mechanism of coalbed methane in soft coal seams and the formulation of practical coalbed methane control measures.

\section{Experimental}

2.1. Experimental Apparatus. The self-developed high/lowtemperature pressure swing adsorption-desorption experimental system is shown in Figure 1. The experimental equipment mainly consists of an adsorption system, a gas desorption system, a refrigeration system, an electrical control system, and a computer data acquisition and processing system. The gas adsorption experiment adopts the pressure range is $0-8 \mathrm{MPa}$. The working temperature is $0-40^{\circ} \mathrm{C}$.

The low-temperature nitrogen adsorption test uses the ASAP-2020 specific surface and pore size analyzer produced by Mircromeritics. The pore volume is less than $0.0001 \mathrm{~cm}^{3} /$ $\mathrm{g}$, the pore size analysis range is 0.35 to $500 \mathrm{~nm}$, and the specific surface area analysis range is 0.0005 to $5000 \mathrm{~m}^{2} / \mathrm{g}$. The pore size classification method defined by the International Union of Pure and Applied Chemistry (referred to as IUPAC) is used. Using the IIGMA scanning electron microscope produced by Zeiss, the acceleration voltage range of the instrument is $0.1-30 \mathrm{kV}$, and the magnification range is $12-500 \mathrm{kX}$. It can analyze the microstructure of powder, block, and film samples.

2.2. Sample Preparations. The soft coal sample used in the test was taken from the Lvtang Coal Mine (LT) in Bijie District, Guizhou, China, and the hard coal used was taken from the Quanlun Coal Mine (QL) in Qinglong County, Xingyi City. The geographical location of the LT and QL is shown in Figure 2.

Each coal sample is not less than $1 \mathrm{~kg}$. According to the China Coal Industry Standard [19] to prepare the coal sample for proximate analysis and gas adsorption experiment, the humidification process for coal samples is shown in Figure 3.

The data of proximate analysis of coal samples, coal hardiness coefficient, and initial gas diffusion velocity of coal are shown in Table 1. Select a small coal sample with a flat cross section with a particle size of 1 to $2 \mathrm{~cm}^{3}$ for low-temperature nitrogen adsorption test. It should be handled with care during operation. Table 1 data display that the firmness coefficient of soft coal is significantly lower than that of hard coal, and the initial rate of dispersal is greater than that of hard coal.

2.3. Experimental Methods. Coal sample gas desorption process simulation was conducted by employing the experimental device shown in Figure 3. The gas desorption environment of the sample was always maintained at a temperature of $30 \pm 1^{\circ} \mathrm{C}$ and a gas outlet pressure of $0.1 \mathrm{MPa}$ during the measurement process, and gas desorption of the coal sample could be considered to be an isothermal and isostatic desorption process. The experiment is conducted following the steps:

(1) The experimental coal sample is loaded into the coal sample tank.

(2) The coal sample tank and the reference tank are fully degassed using a vacuum pump.

(3) When the vacuum in the experimental system reaches $25 \mathrm{kPa}$, gas is introduced into the reference tank. When the gas pressure is stable, the coal sample tank and the reference tank are connected to conduct gas adsorption. 


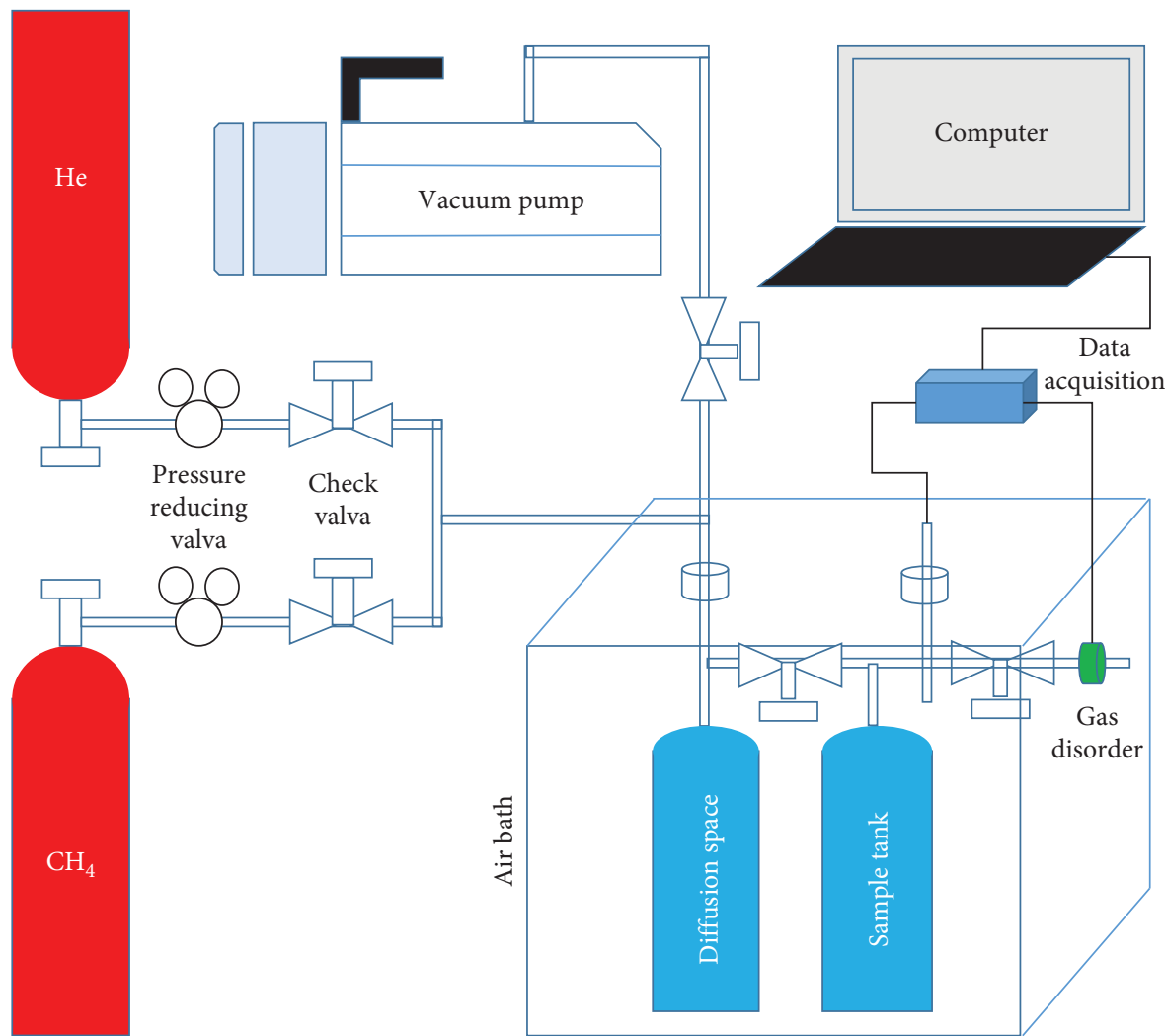

FIGURE 1: Schematic diagram of the experimental platform for the high-low temperature coal gas desorption and adsorption experiments.

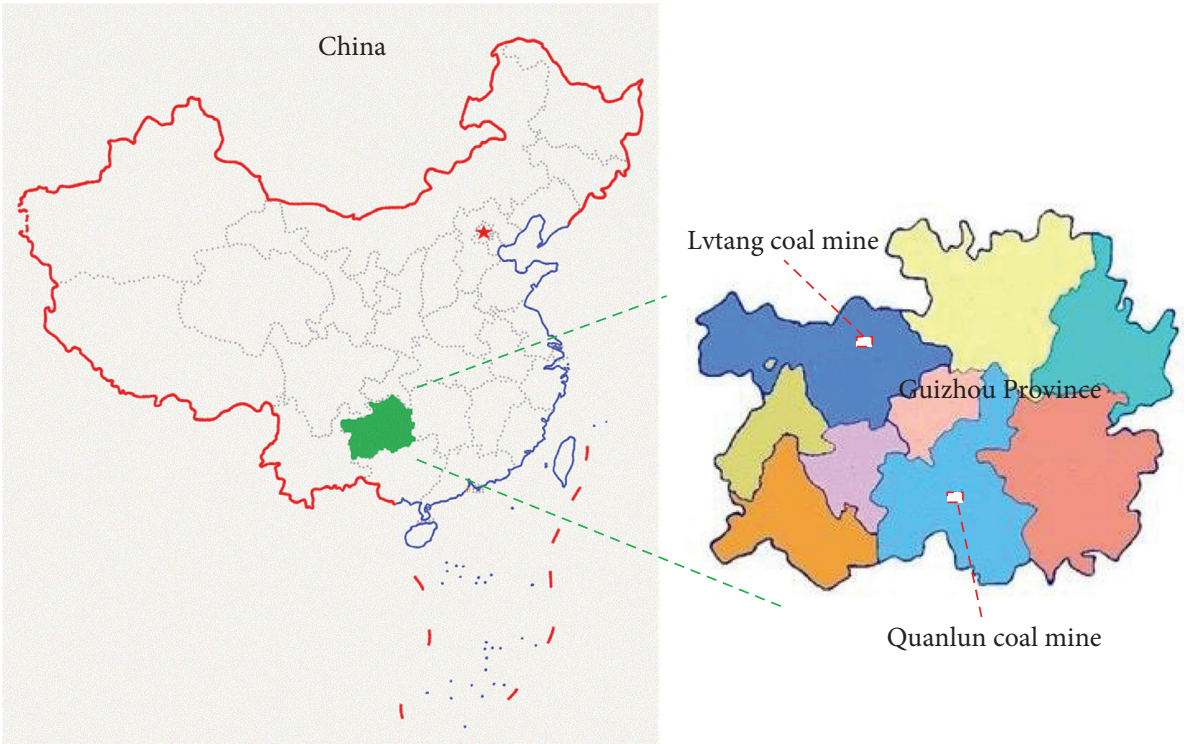

Figure 2: Geographical location of the LT and QL coal mine.

(4) As gas adsorption balance in the coal sample is reached, the gas inlet valve is first closed, and the gas outlet valve is opened second to conduct the gas desorption experiment.

(5) After the experiment is completed, the measured gas desorption amount is converted into volume in the standard state.

\section{Experimental Results}

3.1. Pore Microstructure Analyses. The coal sample from the Lvtang coal mine showed a microscopic breccia structure when observed under a microscope, as shown in Figure 4(a). Its characteristic is that the coal body is broken into particles of different sizes, some of which have more obvious edges and corners. The particles on the coal surface are mostly 


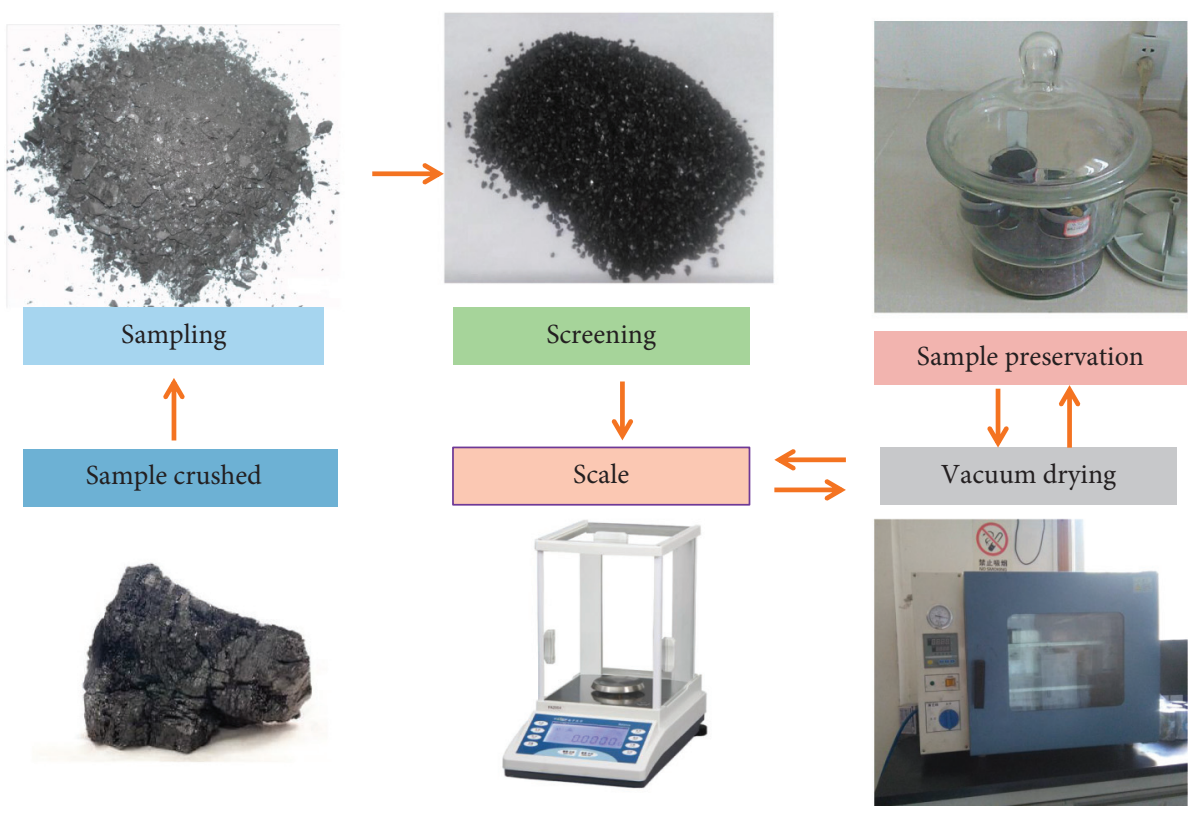

FIgURe 3: Humidification process for the coal samples.

TABLE 1: Experiment and proximate analysis data of coal samples.

\begin{tabular}{lccccccc}
\hline Coal type & Coal mine & Mark as & Mad & Aad & $V$ daf & $f$ & \multicolumn{1}{c}{$\Delta P$} \\
\hline \multirow{2}{*}{ Soft coal } & \multirow{2}{*}{ Lvtang } & LT-1 & 0.73 & 10.98 & 12.1 & 1912 & 19.8 \\
& & LT-2 & 0.83 & 12.1 & 15.1 & 0.1622 & 20.6 \\
\hline \multirow{2}{*}{ Hard coal } & \multirow{2}{*}{ Quanlun } & QL-1 & 6.54 & 7.24 & 36.11 & 0.6742 \\
& & QL-2 & 5.98 & 6.65 & 35.25 & 0.6982 & 11.4 \\
& & & &
\end{tabular}

Mad is moisture content on air-dry (ad) basis, \%; Aad is ash content on air-dry (ad) basis, \%; Vdaf is volatile matter content on dry-ash-free (daf) basis, \%; $f$ is coal hardiness coefficient, dimensionless; $\Delta P$ is the initial gas diffusion velocity of coal, $\mathrm{mmHg}$.

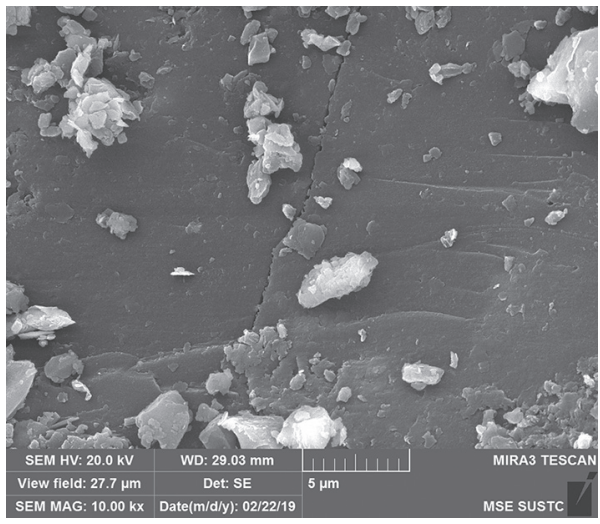

(a)

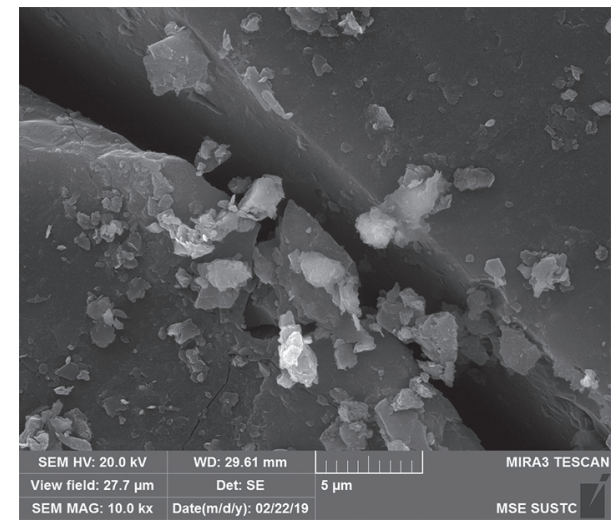

(b)

FIgURE 4: The microstructure of the coal sample. (a) Lvtang coal sample. (b) Quanlun coal sample.

irregular polyhedrons with sharp edges and corners. It shows that the coal sample has poor plasticity and high brittleness. At the same time, it can be seen that a large number of irregular pores are developed on the surface of the coal sample, and the size of the pores is different. The existence and density of the pores indicate that the coal has a certain gas storage capacity. In addition, the existence of a large number of holes reduces the mechanical strength of coal, which is one of the reasons why soft coal is easily broken.

The surface of the coal sample in Quanlun Coal Mine is relatively flat, and a large, dominantly developed fissure can be seen to develop stably in the longitudinal direction. The 
fissure penetrates the entire coal surface in the visible range, as shown in Figure 4(b). The surface of the coal sample is distributed with some inorganic components such as pointlike and pile-like clay materials. On the one hand, the presence of inorganic components increases the strength of the coal and acts as the skeleton of the coal; on the other hand, it destroys the uniformity of the coal and softens when exposed to water, which directly reduces the anti-destructive ability of the coal body itself.

\subsection{Specific Surface Areas and Pore Volume Characteristics.} Two sets of soft coal experiment coal samples from LT and two sets of hard coal experiment coal samples from QL were selected, and a total of 4 sets of nitrogen adsorption tests were carried out. The pore volume characterization method adopts the $\mathrm{BJH}$ method, and the specific surface area characterization method adopts the BET method and the Langmuir method. The pore structure characteristics of the soft and hard coal surface are analyzed from different angles. The results of the low-temperature nitrogen adsorption test are shown in Table 2.

It can be seen from Table 2 that the BET specific surface area values of soft and hard coal are all smaller than the corresponding Langmuir specific surface area values. This is because the calculation methods of the two models are different. The Langmuir model assumes that the gas adsorption on the coal surface is limited to a single layer, while the BET model believes that the gas adsorption on the coal surface can be multilayer adsorption. That is, when the gas reaches the saturated adsorption capacity, the adsorption capacity of the BET model is greater than that of the Langmuir model. In addition, single-layer adsorption capacity referred to by Langmuir theory is the saturated adsorption capacity, while the single-layer adsorption capacity referred to by the BET theory is only a theoretical value. The Langmuir saturated adsorption capacity is not equal to the maximum adsorption capacity, but corresponds to the closed point of the adsorption loop on the adsorption isotherm. The appearance of adsorption loops on the adsorption isotherm indicates that there are mesopores or microporous pores in the test object. However, the adsorption in the mesopores appears as capillary condensation. According to theory of Langmuir, it is an adsorbate liquefaction process and cannot be regarded as an adsorption process. Therefore, the Langmuir theoretical model is not suitable for explaining the adsorption behavior of the adsorbate in the mesopores. For porous media, especially materials with more micropores and mesopores, the BET model is more suitable for characterizing the specific surface area. The BET specific surface area of soft coal ranges from 10.4158 to $14.3245 \mathrm{~m}^{2} / \mathrm{g}$, and the $\mathrm{BJH}$ pore volume ranges from 0.006117 to $0.008416 \mathrm{~cm}^{3} / \mathrm{g}$. The specific surface area and pore volume of soft coal are 2.33 times and 1.79 times larger than those of hard coal, respectively. Specific surface area and pore volume are important indicators to characterize the ability of coal to absorb gas. The larger the value, the larger the gas absorption volume of coal. The average pore diameter of soft coal is smaller than that of hard coal, indicating that the inner surface area of the pores of soft coal is larger in unit volume, so that the pore surface provides more gas adsorption sites.

3.3. Relationship between Adsorption/Desorption Curve and Pore Structure Characteristics. The shape of the different relative pressure sections of the adsorption isotherm reflects the physical characteristics of the coal surface and the relationship between the coal and gas. The relative pressure can evaluate the performance of the coal reservoir, especially the permeability of the coal. The low-temperature nitrogen adsorption/desorption curve of a typical soft and hard coal sample is shown in Figure 5.

It can be seen from Figure 5 that when the relative pressure is zero, the nitrogen adsorption capacity of soft and hard coal is greater than zero. It shows that a certain volume of nitrogen has been adsorbed in the micropores, and the adsorption capacity of soft coal at the starting point is greater than that of hard coal. It can be seen that soft coal has a larger micropore area than hard coal. This is because the gas is preferentially adsorbed in the micropores of the coal. The more micropores contained in the coal, the stronger the superposition of the adsorption potential energy in the micropores. As a result, nitrogen adsorption capacity increases faster. When the relative pressure is between 0 and 0.2 , the adsorption curve shows an upward convex trend. It shows that the force between the surface of the coal body and the nitrogen molecules is strong [20]. When the relative pressure is between 0.5 and 1, soft and hard coal have different adsorption hysteresis loops. And the hysteresis range of soft coal is larger than that of hard coal. The size of the hysteresis ring reflects the connectivity of coal pores. It shows that the pore connectivity of soft coal is better than that of hard coal [21]. Compared with hard coal, the hysteresis loop of soft coal is more obvious in the desorption process. This shows that it is very likely that a large amount of gas will escape in a short time when mining soft coal seams. Major outburst prevention and stress control measures should be taken to mine and ease the pressure during mining. When the relative pressure is higher than 0.9 , the adsorption curve rises sharply, and the rise rate of the soft coal adsorption curve is obviously greater than that of the hard coal adsorption curve. The opening degree of coal pores is related to the rate of rise of the adsorption line. The faster the rise, the greater the opening degree of the pores.

3.4. Fractal Geometric Characteristics of Coal Surface. The fractal dimension can be used to characterize the fractal characteristics of the pore surface of coal, and its numerical value can be obtained from low-temperature nitrogen adsorption test data. Calculating and analyzing from different angles, taking the adsorption capacity at the inspection point, the relationship between fractal dimension and adsorption capacity is as follows [22]:

$$
\ln Q=\left(D_{f}-3\right) \ln \left(\ln \frac{P_{0}}{P}\right)+C,
$$

where $P$ is nitrogen partial pressure, MPa; $Q$ is the adsorption capacity when the equilibrium pressure is $P, \mathrm{~cm}^{3} / \mathrm{g}$; 
TABLE 2: Coal sample nitrogen adsorption test results.

\begin{tabular}{|c|c|c|c|c|c|c|}
\hline $\begin{array}{l}\text { Coal } \\
\text { type }\end{array}$ & $\begin{array}{l}\text { Coal } \\
\text { mine }\end{array}$ & $\begin{array}{l}\text { Mark } \\
\text { as }\end{array}$ & $\begin{array}{l}\text { BET specific surface area } \\
\left(\mathrm{m}^{2} \cdot \mathrm{g}^{-1}\right)\end{array}$ & $\begin{array}{l}\text { Langmuir specific surface area } \\
\qquad\left(\mathrm{m}^{2} \cdot \mathrm{g}^{-1}\right)\end{array}$ & $\begin{array}{l}\text { BJH pore volume } \\
\left(\mathrm{cm}^{3} \cdot \mathrm{g}^{-1}\right)\end{array}$ & $\begin{array}{l}\text { Average pore size } \\
(\mathrm{nm})\end{array}$ \\
\hline \multirow{2}{*}{ Soft coal } & \multirow{2}{*}{ Lvtang } & LT-1 & 12.2148 & 14.2310 & 0.008416 & 6.1773 \\
\hline & & LT-2 & 14.3245 & 16.2412 & 0.007415 & 6.2112 \\
\hline \multirow{2}{*}{$\begin{array}{l}\text { Hard } \\
\text { coal }\end{array}$} & \multirow{2}{*}{ Quanlun } & QL-1 & 6.2271 & 8.1648 & 0.004452 & 14.8725 \\
\hline & & QL-2 & 4.3718 & 6.2147 & 0.003674 & 15.5149 \\
\hline
\end{tabular}

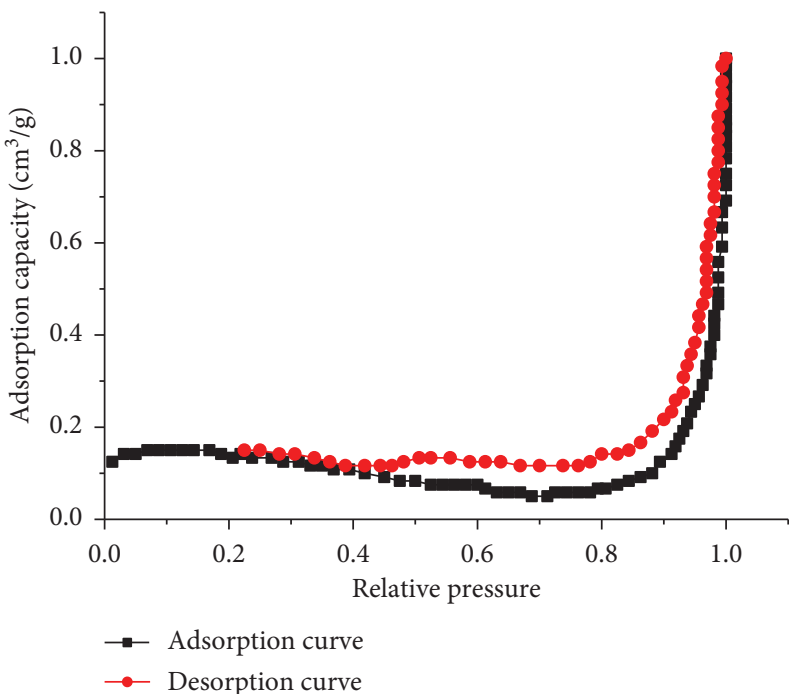

(a)

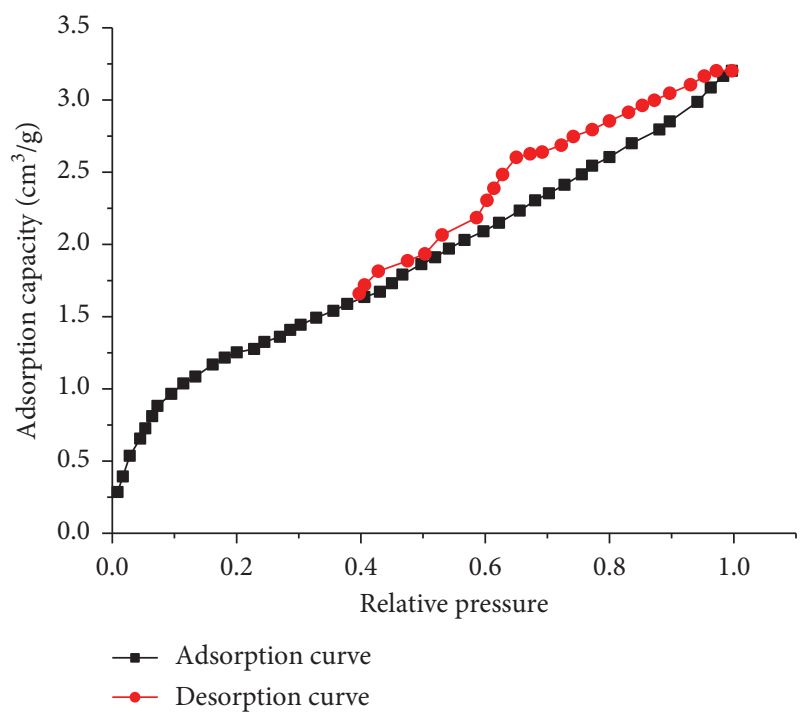

(b)

Figure 5: Low-temperature nitrogen adsorption/desorption curve of coal sample. (a) Soft coal. (b) Hard coal.

$P_{0}$ is saturated vapor pressure of gas adsorption, $\mathrm{MPa} ; D_{f}$ is fractal dimension, which characterizes the fractal characteristics of coal pore surface, dimensionless; and $C$ is constant, dimensionless.

Taking the specific surface area of coal as an investigation point, the relationship between fractal dimension and specific surface area is as follows [23]:

$$
\ln A=\left(D_{f}-2\right) \ln \left(\ln \frac{P_{0}}{P}\right)+C
$$

where $A$ is specific surface area of coal surface, $\mathrm{m}^{2} / \mathrm{g}$; other parameter symbols have the same meaning as above.

In the low-temperature nitrogen adsorption experiment, a straight line can be obtained by plotting $\operatorname{Ln} Q$ and $\operatorname{Ln} A$ against $\operatorname{Ln}\left(\operatorname{Ln} P_{0} / P\right)$, and the fractal dimension can be obtained from the slope of the straight line. According to formula (1) and (2), the size of the fractal dimension of the coal sample surface can be calculated, and the calculation results are shown in Table 3 .

The data in Table 3 shows that the calculation results of formula (1) and (2) are slightly different, and the calculation result of formula (1) is slightly larger than that of formula (2). This difference may be due to the micropores or mesopores in the coal. The difference in the pore size of the micropores or mesopores results in the difference in the adsorption potential of the micropores for nitrogen, so that nitrogen molecules cannot completely and effectively fill all mesopores. The scoring dimension results of the two calculation formulas reflect the characteristics of coal pore structure to a certain extent. It can be seen from Table 3 that the calculation results of the surface fractal dimension of soft coal are between 2.12 and 2.35 , the calculation results of the surface fractal dimension of hard coal are between 1.43 and 1.62, and the average surface fractal dimension of soft coal is 1.47 times that of hard coal. The larger the fractal dimension, the rougher the surface and the larger the specific surface area of coal pores. This also reflects that soft coal has more developed pores than hard coal.

3.5. Characteristics of Coal Gas Adsorption. Constant temperature adsorption experimental data of soft and hard coal are shown in Table 4, and the constant temperature adsorption test curve is shown in Figure 6.

Comprehensive analysis of Table 4 and Figure 6 can be drawn that, under the same experimental conditions, the soft coal adsorption constants $a$ and $b$ are both larger than the hard coal adsorption constants. Soft coal has a larger saturated adsorption capacity than hard coal, and at the same time soft coal reaches the saturated adsorption capacity faster. It shows that the gas adsorbs and stays on the pore surface of the soft coal for a long time, the gas coverage is high, and the soft coal has a stronger ability to adsorb gas. 
TABLE 3: Calculation results of the surface fractal dimensions of coal samples.

\begin{tabular}{lcccc}
\hline Coal mine & Mark as & Relative pressure $P / P_{0}$ range & Formula (1) fractal dimension calculation & Formula (2) fractal dimension calculation \\
\hline \multirow{2}{*}{ Lvtang } & LT-1 & $0.15-0.96$ & 2.34 & 2.23 \\
& LT-2 & $0.17-0.97$ & 2.35 & 2.25 \\
\hline \multirow{2}{*}{ Quanlun } & QL-1 & $0.44-0.99$ & 1.58 & 1.43 \\
& QL-2 & $0.43-0.99$ & 1.62 & 1.55 \\
\hline
\end{tabular}

TABLE 4: Results of constant temperature adsorption experiment of the coal sample.

\begin{tabular}{|c|c|c|c|c|c|}
\hline Coal mine & Mark as & Adsorption constants $a$ & Adsorption constants $b$ & Fitting curve & $R^{2}$ \\
\hline \multirow{2}{*}{ Lvtang } & LT-1 & 18.7513 & 2.6515 & $Q=49.7169 P /(1+2.6515 P)$ & 0.9991 \\
\hline & LT-2 & 18.5424 & 2.5425 & $Q=47.1457 \mathrm{P} /(1+2.5427 P)$ & 0.9992 \\
\hline Quanlun & QL-1 & 18.1 & 0.44 & $Q=7.74 P /(1+0.35 P)$ & \\
\hline
\end{tabular}

The adsorption constants $a$ and adsorption constants $b$ are important indicators of the ability of coal to absorb gas, which conform to the Langmuir adsorption formula: $Q=((a b P) /(1+b P))$. Here, $a$ is the value reflects the maximum adsorption capacity of the coal, $\mathrm{cm}^{3} / \mathrm{g} ; b$ is the value represents the rate at which the coal reaches its saturated adsorption capacity, $\mathrm{MPa}^{-1} ; \mathrm{Q}$ is gas adsorption capacity under a certain pressure, $\mathrm{cm}^{3} ; P$ is the gas pressure of the coal seam, $\mathrm{MPa}$.

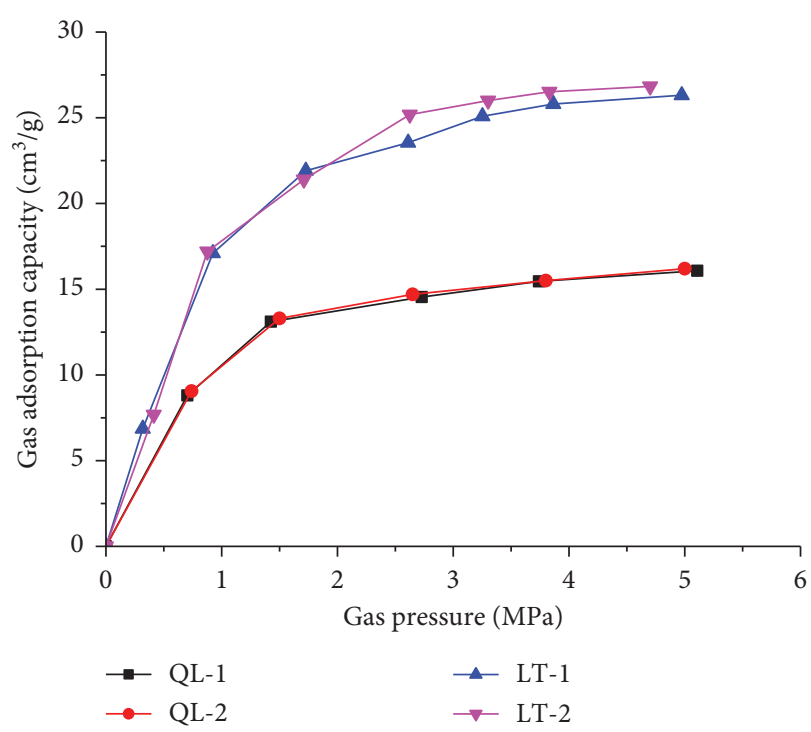

FIGURE 6: Constant temperature adsorption curve of soft and hard coal.

\section{Discussion}

\subsection{The Influence of Pore Type on Gas Adsorption}

4.1.1. The Superposition of Adsorption Potential Energy in the Micropores. The abundant pore structure in the coal body is not only a place for gas storage, but also a channel for gas migration and diffusion. In particular, the presence of micropores greatly increases the surface area of the coal body. Since the size of the micropores is similar to the size of the gas molecules, the gas molecules are surrounded by the micropores. The adsorption force field in the micropores produces a superposition effect, so that the adsorption potential energy is greatly enhanced compared with the adsorption potential energy on the flat pore wall surface. Under low relative pressure, it has stronger adsorption attraction for gas molecules. Studies have shown that the adsorption potential is greatly enhanced in circular pores less than six molecular diameters and in slit pores less than two molecular diameters. The relationship between micropore adsorption volume and adsorption potential energy can be expressed as [24]

$$
\left\{\begin{array}{l}
\varphi=\varphi_{0} \cdot e^{-K \varepsilon^{2}}, \\
\varepsilon=\ln \frac{P}{P_{0}},
\end{array}\right.
$$

where $\varphi$ is adsorption volume, $\mathrm{cm}^{3} ; \varphi_{0}$ is maximum adsorption volume, $\mathrm{cm}^{3} ; K$ is constant related to the micropore structure, dimensionless; and $\varepsilon$ is adsorption potential energy, $J$.

In formula (3), $\varepsilon^{2}$ reflects the superposition of the adsorption potential field on the micropore wall.

4.1.2. Diffusion in Mesopores. The analysis of mesopores is closely related to the nitrogen adsorption/desorption curve, and the specific form of the adsorption hysteresis ring corresponds to different mesopore structures. The large range of the hysteresis ring indicates that the corresponding pores are smooth and the pores have high air permeability, which is conducive to the migration and diffusion of gas. The diffusion of gas in mesopores satisfy Fick diffusion, and the diffusion rate can be expressed as

$$
\frac{\mathrm{d} Q}{\mathrm{~d} t}=-D_{0} \cdot A_{g} \cdot \frac{\mathrm{d} c}{\mathrm{~d} x},
$$

where $\mathrm{dQ} / \mathrm{d} t$ is gas diffusion rate, $\mathrm{kg} / \mathrm{s} ; D_{0}$ is gas diffusion coefficient, $\mathrm{m}^{2} / \mathrm{s} ; A_{\mathrm{g}}$ is the gas diffusion area in the channel, $\mathrm{m}^{2}$; and $\mathrm{d} c / \mathrm{d} x$ is gas concentration gradient, $\mathrm{kg} /\left(\mathrm{m}^{3} \cdot \mathrm{m}\right)$.

In formula (4), “-” means that the direction of gas diffusion in the channel is opposite to the direction of the gas concentration gradient.

4.2. The Effect of Adsorption Residence Time on Gas Adsorption. The time from when the gas molecules hit the surface of the coal body to when they leave the surface and return to the gas phase is called the adsorption residence time. If the adsorption residence time is much longer than 
the molecular vibration period, it can be considered that adsorption has occurred. The inner surface area of the pores of soft coal is larger, it provides more gas adsorption sites, and the adsorption site gas coverage is high. Soft coal has a stronger adsorption potential for gas, and the adsorption residence time of gas on the surface of soft coal is longer than that of hard coal, so the amount of gas adsorption is greater. The gas coverage of coal pore surface can be expressed as

$$
\theta=\frac{a}{a_{m}},
$$

where $\theta$ is the gas coverage on the surface of coal pores, $\left({ }^{\circ}\right) ; a$ is the number of gas molecules adsorbed on the coal surface, unit; and $a_{\mathrm{m}}$ is the number of gas molecules covering the monolayer of $1 \mathrm{~cm}^{2}$ coal surface, unit.

The relationship between the number of gas molecules and the adsorption residence time $\tau$ can be expressed as

$$
\left\{\begin{array}{l}
a=n \cdot \tau, \\
n=\frac{N_{A} P}{(2 \pi M R T)^{1 / 2}},
\end{array}\right.
$$

where $n$ is the number of gas molecules that collide on the surface of the coal body in a unit time, unit; $N_{A}$ is Avogadro's constant, which is $6.02 \times 10^{23} \mathrm{~mol}^{-1}$; $P$ is gas pressure, $\mathrm{MPa}$; $M$ is the relative molecular mass of adsorption, dimensionless; $R$ is the ideal gas constant, which is $8.314 \mathrm{~J} /(\mathrm{mol} \cdot \mathrm{K})$; $T$ is the thermodynamic temperature, $K$; and $\tau$ is adsorption residence time, $S$.

\subsection{The Influence of Fractal Characteristics on Gas Adsorption.} The difference in the fractal characteristics of the coal surface reflects the change of the coal pore structure. The pore size of the soft coal gradually decreases with the increase of the fractal dimension of the coal surface. The change curve of the calculation result using formula (1) is shown in Figure 7.

The larger the fractal dimension, the higher the surface roughness of the coal and the increase of pores per unit volume. The radius of the pore channel decreases, which increases the pore volume and specific surface area. The gas adsorption sites provided by the pore surface of the coal increase, and the gas adsorption capacity is enhanced.

According to the theory of fractal geometry, there is the following relationship between the pore radius and the cumulative number in coal [25]:

$$
\left\{\begin{array}{l}
N \infty r^{-D_{f}}, \\
N=A_{2} \cdot r_{m}^{D_{f}} \cdot\left(\lambda^{D_{f}}-1\right) \cdot\left(r_{0}^{-D_{f}}-r_{m}^{D_{f}}\right) \\
\lambda=\frac{r_{0}}{r_{m}},
\end{array}\right.
$$

where $N$ is the total number of pores per unit volume of coal, unit; $r$ is the radius of the pores in the coal, $\mathrm{nm} ; A_{f}$ is does not significantly include the proportional coefficient of $D_{f}, r_{m}$ is maximum radius of the pore, $\mathrm{nm} ; r_{0}$ is minimum radius of

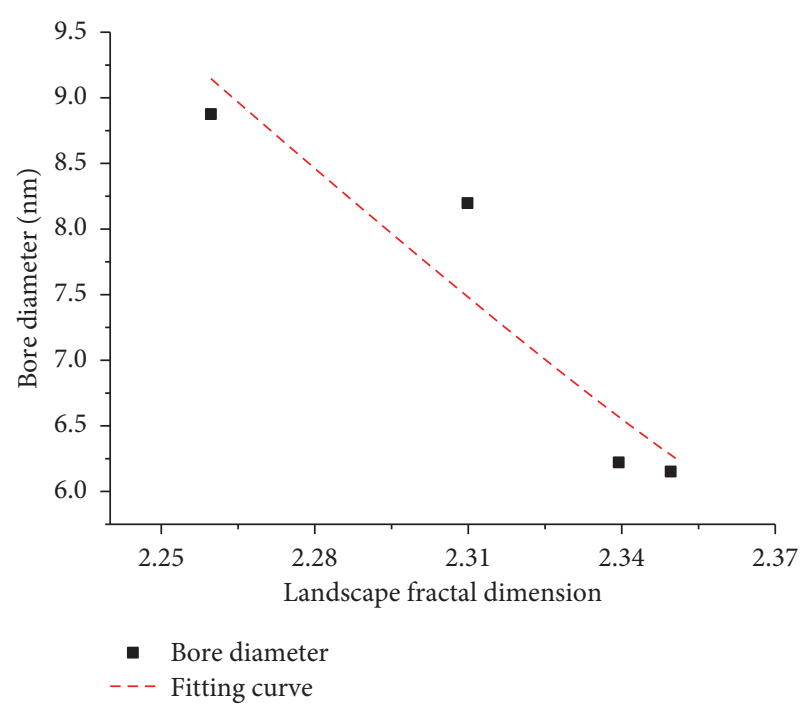

FIgURE 7: The relationship curves between aperture and fractal dimension.

pore, $\mathrm{nm}$; and $\lambda$ is the ratio of the minimum and maximum pore radius.

From formula (7), the distribution density function of the pore radius in the coal body can be obtained:

$$
Y(r)=A D_{f} r^{-\left(1+D_{f}\right)} \text {. }
$$

From formula (8), the average radius of pores in the coal body can be obtained as

$$
\bar{r}=\frac{D_{f}}{1-D_{f}} r_{m} \frac{1-\lambda^{1-D_{f}}}{\lambda^{-D_{f}}-1} .
$$

Analyzing formula (9), it can be obtained that the average radius of coal pores depends entirely on the fractal dimension $D_{f}$ of the coal, the upper and lower limits $r_{m}$ and $r_{0}$ of the pore distribution in the coal, and the total number of pores in the porous medium per unit volume $N$.

In addition, upper and lower limits $r_{m}$ and $r_{0}$ of the pore distribution in the coal body and the total number $N$ of pores per unit volume of porous media are constant. Then, the average radius $\bar{r}$ of the pore gradually decreases with the increase of the fractal dimension $D_{f}$.

\section{Conclusion}

In this paper, a self-developed adsorption/desorption device is used to test the gas adsorption and desorption performance of soft coal and hard coal under low-temperature conditions, combined with scanning electron microscope and low-temperature nitrogen adsorption experiment to test the pore characteristics of experimental soft coal and hard coal. Analyze the microscopic pore and fracture structure and connectivity of typical soft coal, use the box dimension algorithm to measure the fractal dimension of the microstructure of the coal sample, and compare and analyze the relevant characteristics of hard coal. And we got the following conclusions: 
(1) The results of the low-temperature nitrogen adsorption test calculated that the BET specific surface area of soft coal ranges from 10.4158 to $14.3245 \mathrm{~m}^{2} / \mathrm{g}$, and the $\mathrm{BJH}$ pore volume ranges from 0.006117 to $0.008416 \mathrm{~cm}^{3} / \mathrm{g}$, both of which are larger than those of hard coal. It shows that soft coal has more pores than hard coal, and the inner surface of the pores is larger. Particularly, the number of micropores and the superposition of the adsorption potential energy existing in the micropores make the adsorption potential energy of soft coal to gas greater.

(2) According to the nitrogen adsorption test data, the fractal characteristics of the pore surface of soft and hard coal are calculated, and the average fractal dimension of the surface of soft coal is more than double that of hard coal. It shows that the surface roughness of soft coal is high, there are many pores per unit volume, and the inner surface of the pores can provide more adsorption sites. The gas adsorption site coverage on the surface of the soft coal body is high, and the adsorption residence time is long. Therefore, soft coal has a stronger ability to absorb gas than hard coal.

(3) Compared with hard coal, soft coal exhibits a larger adsorption hysteresis loop in the nitrogen adsorption test. It shows that the pore connectivity of coal is better than that of hard coal. When mining soft coal seams, it is more likely to cause a large amount of gas escape in a short time. When mining, the main prevention and control measures should be taken to extract and relieve pressure [26].

\section{Data Availability}

All the data generated or published during the study are included within the article; no other data were used to support this study.

\section{Conflicts of Interest}

The authors declare that they have no conflicts of interest regarding the publication of this paper.

\section{Acknowledgments}

This work was supported by the Guizhou Province Science and Technology Support Program Funding Project ([2021] General 349), the project supported by an Open-End Fund of the Work Safety Key Lab on Prevention and Control of Gas and Roof Disasters for Southern Coal Mines (E21822), and a youth project supported by the National Natural Science Foundation of China (51804114).

\section{References}

[1] L. Bing, Z. Guocheng, S. Zhimin, and R. Jiangang, "Analysis on coupling relationship between pore structure of deformed coal and $\mathrm{CH}_{4}$ adsorption-desorption characteristics,"
International Journal of Earth Sciences and Engineering, vol. 7, no. 1, pp. 90-98, 2014.

[2] G. Xue, H. Liu, and W. Li, "Deformed coal types and pore characteristics in hancheng coalmines in eastern weibei coalfields," International Journal of Mining Science \& Technology, vol. 22, no. 5, pp. 681-686, 2012.

[3] Q. Yuan, X. L. Ge, S. P. Zhou, and R. G. Deng, "Study on the variation of fractal and mutifractal characteristics of coal particles in the ultra-fine pulverizing process," Advanced Materials Research, vol. 347-353, pp. 3653-3657, 2011.

[4] T. Yin, D. Liu, Y. Cai, Y. Zhou, and Y. Yao, "Size distribution and fractal characteristics of coal pores through nuclear magnetic resonance cryoporometry," Energy \& Fuels, vol. 31, no. 8, pp. 7746-7757, 2017.

[5] C. Sitprasert, Z. H. Zhu, F. Y. Wang, and V. Rudolph, "A multi-scale approach to the physical adsorption in slit pores," Chemical Engineering Science, vol. 66, no. 22, pp. 5447-5458, 2011.

[6] S. P. Rigby, R. S. Fletcher, and S. N. Riley, "Characterisation of porous solids using integrated nitrogen sorption and mercury porosimetry," Chemical Engineering Science, vol. 59, no. 1, pp. 41-51, 2004.

[7] X. Zhang, H. Yao, W. Li, X. Wang, and J. Yan, "Fractal characteristics of nano-scale pore structure of structural coal in Hancheng mining area," Coal Geology and Prospecting, vol. 42 , no. 5, pp. 5-8, 2014.

[8] S. An, S. Sang, Y. Li et al., "Pore fractal characteristics of high coal-grade reservoirs in the southern Qinshui Basin," China Coal Geology, vol. 23, no. 2, pp. 17-21, 2011.

[9] B. Jiang, B. Lin, H. Wu et al., "Study on the characteristics of ultramicro pore structure and fractal law of coal and rock," Journal of Hunan University of Science and Technology, vol. 25, no. 3, pp. 15-18, 2010.

[10] G. Wang, Y. Qin, J. Shen et al., "Dynamic-change laws of the porosity and permeability of low-to medium-rank coals under heating and pressurization treatments in the eastern junggar basin, China," Journal of Earth Science, vol. 29, no. 3, pp. 133-141, 2018.

[11] A. Y. Klimenko, D. N. Saulov, and P. Massarotto, "Conditional model for sorption in porous media with fractal properties," Transport Porous Media, vol. 92, pp. 745-765, 2012.

[12] D. Kong, S. Pu, Z. Cheng et al., "Coordinated deformation mechanism of the top coal and filling body of gob-side entry retaining in a fully mechanized caving face," International Journal of Geomechanics, vol. 21, no. 4, Article ID 04021030, 2021.

[13] Y. Yao, D. Liu, D. Tang et al., "Fractal characterization of adsorption - pores of coals from north: an investigation on $\mathrm{CH}_{4}$ adsorption capacity of coals," International Journal of Coal Geology, vol. 73, pp. 27-42, 2008.

[14] S. Harpalant and G. L. Chen, "Influence of gas production induced volumetric strain on permeability of coal," Geotechnical and Geological Engineering, vol. 15, no. 4, pp. 303325, 1997.

[15] H. GaoM. Masakutsan et al., "Statical distribution characteristics of pyriding transport in coal particales and a series of new phenomenological models for overshoot and nonovershoot solvent swelling of coap particals," Energy and Fuels, vol. 13, no. 2, pp. 518-528, 1999.

[16] S. Zhou and B. Lin, Theory of Coal Seam Gas Occurrence and Flow, Coal Industry Press, Beijing, China, 1999. 
[17] C. O. Karacan, "Heterogeneous sorption and swelling in a confined and stressed coal during $\mathrm{CO}_{2}$ injection," Energ and Fuels, vol. 17, no. 6, pp. 1595-1608, 2003.

[18] C. O. Karacan, "Swelling-induced volumetric strains internal to a stressed coal associated with $\mathrm{CO}_{2}$ sorption," International Journal of Coal Geology, vol. 72, no. 3-4, pp. 209-220, 2007.

[19] F. Wang, Y. Liang, X. Li et al., "Orthogonal experimental study on multifactor conditions for gas desorption in coal," Advances in Civil Engineering, vol. 2019, pp. 1-12, 2019.

[20] A. D. Swann, "Analytical solutions for determining naturally fractured reservoir properties by well testing," Society of Petroleum Engineers Journal, vol. 16, no. 3, pp. 117-122, 1976.

[21] J. E. Warren, P. J. Root, "The behavior of naturally fractured reservoirs," Society of Petroleum Engineering, vol. 3, no. 3, pp. 245-255, 1963.

[22] G. I. Barenblatt, I. P. Zheltov, and I. N. Kochina, "Basic concepts in the theory of seepage of homogeneous liquids in fissured rocks [strata]," Journal of Applied Mathematics \& Mechanics, vol. 24, no. 5, pp. 1286-1303, 1960.

[23] K. Jeffrey, J. Jacek, M. Sharon, and P-R. Javier, "Unified method to the total pore volume and pore size distribution of hierarchical zeolites from argon adsorption and mercury intrusion," Langmuir, vol. 31, no. 4, pp. 1242-1247, 2015.

[24] J. Sobczyk, "A comparison of the influence of adsorbed gases on gas stresses leading to coal and gas outburst," Fuel, vol. 115, no. 1, pp. 288-294, 2014.

[25] E. B. Louw, G. D. Mitchell, J. Wang et al., "Constitution of drop-tube generated coal chars from vitrinite-rich and inertinite-rich South African coals," Energy \& Fuels, vol. 30, no. 1, pp. 112-120, 2016.

[26] L. Han, Y. Lin, and K. Chen, "Proximate analysis of coal," National standards of People's Republic of China, vol. 99, pp. 1-9, 2008. 